\title{
Differential Roles of Accumbal GSK3 $\beta$ in Cocaine versus Morphine-Induced Place Preference, U50,488H-Induced Place Aversion, and Object Memory
}

\author{
Xiangdang Shi, ${ }^{1}$ Jeffrey L. Barr, ${ }^{1}$ Eva von Weltin, Cassandra Wolsh, and Ellen M. Unterwald \\ Center for Substance Abuse Research and Department of Pharmacology, Lewis Katz School of Medicine at Temple University, \\ Philadelphia, Pennsylvania
}

Received April 19, 2019; accepted July 31, 2019

\begin{abstract}
Previous research has demonstrated that activity of glycogen synthase kinase-3 (GSK3) is necessary for the rewarding effects of cocaine. In the present study, a conditional GSK3 $\beta$ gene knockdown model was used to determine if GSK3 $\beta$ activity specifically in the nucleus accumbens is important for cocaine conditioned reward. The roles of accumbal GSK3 $\beta$ in morphine conditioned reward, trans-( \pm )-3,4-dichloro-N-methyl-N-[2-(1pyrrolidinyl)cyclohexyl]benzeneacetamide methanesulfonate salt (U50,488H)-induced conditioned place aversion, and cognitive function were also studied. Adult male and female GSK $3 \beta$-floxed or wild-type mice were injected with adeno-associated virus/Cre into the nucleus accumbens to reduce expression of GSK3 $\beta$ and underwent behavioral testing 4 weeks later. The development of cocaine-induced conditioned place preference was significantly attenuated in mice with reduced levels of GSK3 $\beta$ in the nucleus accumbens, whereas the development of morphine-induced place preference remained intact. Conditional knockdown of GSK $3 \beta$ in the accumbens prevented the development of conditioned aversion produced by $U 50,488 \mathrm{H}$, a $\kappa$-opioid receptor agonist. Cognitive memory tests revealed deficits in
\end{abstract}

object location memory, but not novel object recognition in mice with accumbal GSK3 $\beta$ knockdown. These data demonstrate that GSK3 $\beta$ in the nucleus accumbens is required for cocaine conditioned place preference and $\mathrm{U} 50,488 \mathrm{H}$ conditioned place aversion, as well as spatial memory in object location task, indicating differential roles of GSK3 $\beta$ in the psychostimulant and opiate reward process, as well as in memory for spatial locations and object identity.

\section{SIGNIFICANCE STATEMENT}

Knockdown of GSK3 $\beta$ in the nucleus accumbens attenuated the development of cocaine-induced place preference, as well as conditioned place aversion to $U 50,488 \mathrm{H}$, a $\kappa$-opioid receptor agonist. In contrast, the development of morphine place preference was not altered by GSK3 $\beta$ knockdown. GSK3 $\beta$ knockdown in nucleus accumbens impaired performance in the object location task, but not the novel object recognition task. These results elucidate different physiological roles of accumbal GSK $\beta$ in conditioned reward, aversion, and memory.

\section{Introduction}

The importance of glycogen synthase kinase-3 (GSK3) function in the pathology of neuropsychiatric disorders including schizophrenia, bipolar disorder, and drug addiction has been recognized (Grimes and Jope, 2001; Gould et al., 2004; Lovestone et al., 2007; Perrine et al., 2008). Two isoforms of GSK3, GSK3 $\alpha$ and GSK3 $\beta$, exist. GSK3 $\beta$ is the major isoform in the brain and is highly abundant in the hippocampus, cortex, and striatum (Yao et al., 2002; Jope and Johnson, 2004). GSK3 $\beta$ activity is tightly regulated, with the major regulatory mechanism being the phosphorylation of an N-terminal serine, Ser9-GSK3 $\beta$. GSK3 $\beta$ is a constitutively

This work was supported by the National Institutes of Health [Grants R01 DA043988 (to E.M.U.) and P30 DA013429 (to E.M.U.)]. The authors declare that they have no conflicts of interest.

${ }^{1}$ X.S. and J.L.B. contributed equally to this work.

https://doi.org/10.1124/jpet.119.259283. active kinase, and kinase activity is inhibited by phosphorylation of Ser9-GSK3 $\beta$ (Jope and Johnson, 2004; Kockeritz et al., 2006).

GSK3 $\beta$ is an important mediator of dopaminergic neurotransmission and the expression of dopamine-associated behaviors (Beaulieu et al., 2005, 2011). For example, elevated synaptic dopamine results in activation of GSK3 $\beta$ in the striatum of mice with genetic deletion of the dopamine transporter, and GSK3 inhibition reduces the hyperactive phenotype of these mice (Beaulieu et al., 2004, 2005). Similarly, previous reports from our laboratory have demonstrated that GSK3 $\beta$ activity in the nucleus accumbens is enhanced after acute cocaine exposure (Perrine et al., 2008; Miller et al., 2014), and dopaminergic D1 and D2 receptors as well as glutamatergic $N$-methyl-D-aspartate receptors contribute to the activation of GSK3 $\beta$ by cocaine (Miller et al., 2014). Activation of GSK3 is required for the rewarding effects of cocaine. Systemic administration of GSK3 inhibitors prior

ABBREVIATIONS: AAV, adeno-associated virus; DI, discrimination index; GSK3, glycogen synthase kinase-3; flx, floxed; MSN, medium spiny neuron; U50, 488H, trans-( \pm )-3,4-dichloro-N-methyl-N-[2-(1-pyrrolidinyl)cyclohexyl]benzeneacetamide methanesulfonate salt; VTA, ventral tegmental area; WT, wild type. 
to cocaine or amphetamine conditioning blocks the development of place preference (Miller et al., 2014; Wickens et al., 2017). Although the requirement of GSK3 activity for the rewarding effects of psychostimulants has been established, the specific brain region(s) and GSK3 isoform contributing to this mechanism has yet to be determined. Furthermore, there are no reports indicating whether GSK3 $\beta$ is necessary for the conditioned rewarding or aversive effects of opioids.

Current pharmacological approaches cannot differentiate between GSK3 $\alpha$ and $\beta$ isoforms because the inhibitors, while selective for GSK3, are not selective for the individual isoforms (Coghlan et al., 2000). GSK3 $\beta$ knockout mice are embryonically lethal, and global heterozygous GSK3 $\beta$ knockout induces long-term spatial memory impairment (Kimura et al., 2008). Because of the limitations to these other approaches, the present study used a conditional knockdown approach to specifically study GSK3 $\beta$ in the nucleus accumbens of adult female and male mice. Using this genetic conditional knockdown model, the role of accumbal GSK3 $\beta$ in cocaine reward was investigated. This study also determined the importance of GSK $3 \beta$ in morphine conditioned reward, conditioned aversion produced by trans-( \pm )-3,4-dichloro-N-methyl-N-[2(1-pyrrolidinyl)cyclohexyl]benzeneacetamide methanesulfonate salt (U50,488H, a $\kappa$-opioid receptor agonist), and memory for novel objects and object location. The results presented herein indicate that GSK3 $\beta$ in the nucleus accumbens had different roles in cocaine and morphine conditioned reward. Unexpectedly, GSK3 $\beta$ in the nucleus accumbens also contributed to conditioned place aversion produced by a $\kappa$-opioid receptor agonist and to object spatial memory.

\section{Materials and Methods}

\section{Floxed GSK3 $\beta$ Mice}

Floxed (flx) GSK3 $\beta$ flx/flx mice were generously provided by Dr. James Woodgett (Lunenfeld-Tanenbaum Research Institute, Mount Sinai Hospital, Toronto, Canada) (Kockeritz et al., 2006; Patel et al., 2008). Breeding and genotyping were carried out in core resources supported by our Center on Intersystem Regulation by Drugs of Abuse (under Grant P30 DA013429, to Ellen M. Unterwald, principal investigator). Wild-type (WT) littermates were used as controls. After weaning, mice were housed in single sex groups of four or five per cage without additional enrichment objects. Food and water were available ad libitum and mice were maintained on a 12-hour light/dark cycle (7: $00 \mathrm{AM} / 7: 00 \mathrm{PM})$. Mice were handled and weighed daily for 5 days before beginning behavioral testing. Behavioral testing occurred between 9:00 AM and 5:00 PM. Animal use was conducted with an approved protocol from the Temple University Institutional Animal Care and Use Committee and was consistent with the National Institutes of Health Guidelines for the Care and Use of Laboratory Animals.

Delivery of Adeno-Associated Virus into the Nucleus Accumbens. Mice between the ages of 8-10 weeks were placed within a stereotaxic apparatus (Stoelting Co., Wood Dale, IL) while under isoflurane anesthesia. The stereotaxic coordinates used for viral infusion into the nucleus accumbens were: anterior-posterior $+1.4 \mathrm{~mm}$ anterior and $\pm 0.6 \mathrm{~mm}$ lateral from bregma and $-4.2 \mathrm{~mm}$ ventral (Paxinos and Franklin, 2001). A 26-gauge syringe (Hamilton Company, Reno, NV) was inserted through the incision site and delivered $0.5 \mu \mathrm{l}$ of virus [adeno-associated virus (AAV) 1-Cre-Green Fluorescent Protein (GFP) or AAV-GFP, cytomegalovirus promotor, $1.48+13 \mathrm{VG} / \mathrm{ml}$; Signagen Laboratories, Rockville, MD] at $0.1 \mu \mathrm{l} / \mathrm{min}$ with an infusion pump (Harvard Apparatus, Holliston, MA). After
5 minutes, the syringe needle was withdrawn and the infusion was repeated in the opposite hemisphere. Behavioral testing began 4 weeks following viral delivery. The GFP fluorescence was used to assess the accuracy of the bilateral nucleus accumbens infusions. The data from mice with one or both missed placements were removed from the analysis.

\section{Drugs}

Cocaine hydrochloride, morphine sulfate, and U50,488H were generously supplied by the National Institute on Drug Abuse's drug supply program, dissolved in sterile saline, and injected in a volume of $3 \mathrm{ml} / \mathrm{kg}$ (cocaine, i.p.) or $5 \mathrm{ml} / \mathrm{kg}$ (morphine, s.c.) body weight. An equal volume of saline served as the vehicle control.

\section{Experimental Procedures}

Conditioned Place Preference and Aversion. The procedures for conditioned place preference and conditioned place aversion were similar to those described in our prior publication (Miller et al., 2014) and by Liu et al. (2019). Place conditioning occurred in rectangular plastic chambers $(45 \times 20 \times 20 \mathrm{~cm})$ consisting of two unique compartments, one with white and black vertical striped walls and smooth flooring and the other with white walls with black circles and rough flooring. The two compartments were separated by a removable wall/door. Illumination in both compartments was equal and mice had no initial preference to either compartment. As such, a randomized, counterbalanced, unbiased conditioning procedure was used.

Cocaine-induced conditioned place preference. Mice were injected with cocaine $(10 \mathrm{mg} / \mathrm{kg}$, i.p.) or saline, and immediately confined to one compartment of the conditioning chamber where they remained for 30 minutes. Conditioning occurred once per day for four consecutive days, resulting in two conditioning sessions with saline in one compartment and two sessions with cocaine in the opposite compartment of the conditioning chamber. The test for place preference occurred on day 5, when mice had access to both compartments for 30 minutes in a drug-free state. The time spent in each compartment was recorded. Preference scores (reported in seconds) were calculated as follows: (time spent in the cocaine-paired compartment) minus (time spent in the saline-paired compartment).

Morphine-induced conditioned place preference. Mice were injected with saline or morphine $(5 \mathrm{mg} / \mathrm{kg}$, s.c. $)$ and placed into one of the two compartments for 40 minutes. Conditioning occurred two times each day (9:00 AM and 3:00 PM) for 4 days, resulting in four place pairings with each morphine and saline. The test for place preference occurred on day 5 , when mice were placed in the chamber for 20 minutes and allowed to explore both compartments. The amount of time spent in each compartment was measured, and preference scores were calculated as described previously.

U50,488H-induced conditioned place aversion. The procedure was performed as reported by Liu et al. (2019) with slight modification. Mice remained in their home cage for 15 minutes after receiving an injection of saline or U50,488H $(2.5 \mathrm{mg} / \mathrm{kg}$, s.c. $)$, after which they were placed into one of the two compartments of the conditioning chamber for 30 minutes. Mice were conditioned twice daily, once with $\mathrm{U} 50,488 \mathrm{H}$ and once with saline (9:00 AM and 3:00 PM) for 4 days. Place aversion was tested in a drug-free state on day 5 , when mice were allowed to freely move about both compartments for 15 minutes. The time spent in each compartment was recorded and a place aversion score was calculated as follows: (time in U50,488H-paired compartment) minus (time in saline-paired compartment).

Novel object recognition test. This test was used to evaluate memory function and was performed as reported by Silverman et al. (2013). The experiment consisted of three phases: habituation, familiarization, and test phase. On the first day (day 1, habituation phase), each mouse was placed into an empty open-field chamber for 30 minutes without objects. The open-field apparatus is a plastic chamber $(25 \times 20.5 \times 30 \mathrm{~cm})$ with a smooth floor and four nontransparent red walls. On the second day (day 2, familiarization 
phase), each mouse was placed individually into the open-field chamber for 10 minutes, in which the chamber contained two identical sample objects $(\mathrm{A}+\mathrm{A})$. After the familiarization session, mice were placed in a clean version of their home cage with bedding only (no enrichment), one mouse per cage. The open-field arena and objects were cleaned thoroughly with $70 \%$ ethanol after each session. One familiar object and one novel object $(\mathrm{A}+\mathrm{B})$ were placed in the arena in the same location where the two identical objects were located during the familiarization phase. After a 30-minute delay at the end of the familiarization session, the recognition test occurred. Individual mice were place back into the open-field arena with the familiar and novel objects for 10 minutes. The familiarization session and the recognition test were recorded by an overhead camera for subsequent analysis. Object investigation was defined as time spent sniffing the object with the nose pointed toward the object at a distance $\leq 1 \mathrm{~cm}$. Exploration of the two objects (in seconds) during training and test sessions was measured by an experimenter blinded to experimental group. Recognition memory was defined as significantly more time spent investigating the novel versus the familiar object. A discrimination index (DI) was defined as follows: (time exploring the novel object - time exploring the familiar object)/[(time exploring both objects $) \times 100]$.

Object location memory test. The procedures for the object location test were the same as the novel object recognition test described previously with the following exception: identical copies of the sample objects were presented during the test phase but one of the two objects was placed in a new location. A DI was calculated as follows: (time exploring the novel location of the object - time exploring the familiar location of the object)/[(time exploring both objects $) \times 100]$. The same mice were used in both memory tasks with 1 week separating the two tests. Unique sets of objects were used in the two tasks.

Immunofluorescence. Immunohistochemistry with dual fluorescent labeling was used to measure knockdown of GSK3 $\beta$ and confirm injection site. After behavioral testing was completed, anesthetized mice were perfused with $4 \%$ paraformaldehyde and brains were postfixed for 18 hours. Vibratome sections $(40 \mu \mathrm{m})$ spanning the entire nucleus accumbens were obtained and incubated in goat antiGFP (1:500, ab6673; Abcam) and rabbit anti-GSK3 $\beta$ (1:1000, D5C5Z $\mathrm{XP}$ mAb \#12456; Cell Signaling Technology) primary antibodies at $4^{\circ} \mathrm{C}$ for 24 hours. After rinsing the sections in 0.1 M PBS, they were incubated with donkey anti-goat (1:500, Alexa Fluor 488; Invitrogen) and donkey anti-rabbit (1:500, Alexa Fluor 594; Invitrogen) secondary antibodies at room temperature for 2 hours. Sections from WT and GSK3 $\beta$ floxed mice were processed at the same time. Determination of the spatial distribution of AAV-infected nucleus accumbens neurons was made by visualization of labeling. To measure the changes in GSK3 $\beta$ immunoreactivity, brain sections were imaged using a Nikon epifluorescent microscope with an analog camera and immunoreactive product measured using a bioquantification software system (TCW 98; Bioquant, Nashville, TN), as previously described by our group (Miller et al., 2014). Briefly, WT mice were used as control to define threshold of staining. Threshold values were determined based on mean levels of GSK3 $\beta$ immunostaining. The threshold values were used for consistent auto-measurement of all brain sections. The level of GSK3 $\beta$ immunostaining was calculated by dividing the area containing pixels at or above the defined background threshold by the area of the total number of pixels in the chosen field, which was multiplied by 100 to yield the percentage of staining.

Time Line of Experimental Procedures. Figure 1 outlines the timing of the experimental procedures and treatment groups.

\section{Data Analysis}

Conditioned place preference and aversion data were analyzed by one-way ANOVA (for three groups) or two-tailed $t$ test (for two groups). The relationships between the extent of GSK3 $\beta$ knockdown in nucleus accumbens and place preference score were analyzed using correlation analysis (Pearson's test). Novel object recognition and object location tasks were analyzed by two-way ANOVA with object (or location) and
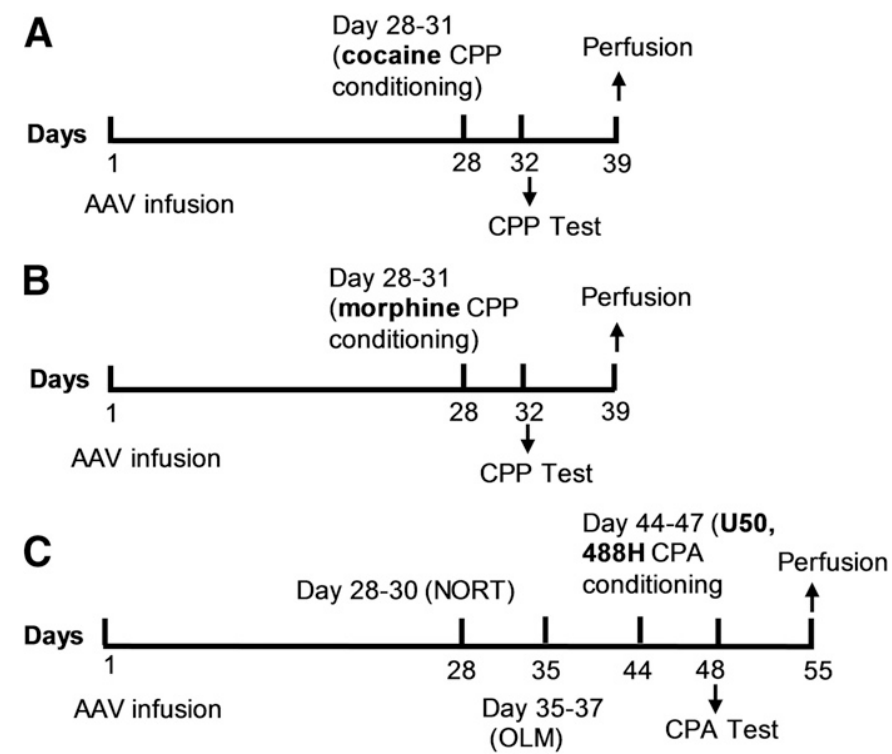

Fig. 1. Time lines of experimental procedures. (A) WT and GSK3 $\beta \mathrm{flx} / \mathrm{flx}$ mice were injected with AAV-Cre-GFP, and GSK3 $\beta$ flx/flx mice were injected with AAV-GFP bilaterally into the nucleus accumbens on day 1 . Mice underwent place conditioning with cocaine $(10 \mathrm{mg} / \mathrm{kg}$, i.p.) using a 4day conditioning procedure. On day 5 (day 32), preference scores were determined during a 30-minute test session. (B) WT and GSK3 $\beta$ flx/flx mice were injected with AAV-Cre-GFP bilaterally into the nucleus accumbens on day 1 . Mice underwent place conditioning with morphine ( 5 or $10 \mathrm{mg} / \mathrm{kg}$, s.c.) using a 4-day conditioning procedure. On test day (day 32 ), preference scores were determined during a 20-minute test session. (C) WT and GSK3 $\beta$ flx/flx mice were injected with AAV-Cre-GFP bilaterally into the nucleus accumbens on day 1 . Novel-object recognition (NORT) and object location memory (OLM) tests were carried out with a 1-week interval between each of the tests. Mice underwent place conditioning with $\mathrm{U} 50,488 \mathrm{H}(2.5 \mathrm{mg} / \mathrm{kg}$, s.c. $)$ using a 4-day conditioning procedure. On test day (day 48), preference scores were determined during a 15-minute test session. Following behavioral tests, all mouse brains were analyzed for efficiency of GSK3 $\beta$ knockdown.

genotype factors followed by Bonferroni's test for multiple comparisons. Discrimination index data were analyzed by one-way ANOVA followed by Bonferroni post tests. Two-way ANOVAs with main factors of genotype and sex were used to assess potential sex differences. GraphPad Prism 4 (La Jolla, CA) was used for statistical analyses.

\section{Results}

Verification of GSK-3 $\beta$ Knockdown in the Nucleus Accumbens. Brains were obtained 5-7 weeks after AAV injection, following behavioral testing, for immunohistochemical analysis of GSK3 $\beta$ levels and confirmation of viral injection location. Three animals were removed from the study due to missed placements. Figure 2 shows representative images of immunostaining against GSK3 $\beta$ in the accumbens from WT (Fig. 2A) and GSK3 $\beta$ flx/flx (Fig. 2B) mice. As can be seen in the magnified images $(40 \times)$ in Fig. 2 , cells in GSK3 $\beta$ flx/flx mice infected with AAV-Cre-GFP (Fig. 2B) had no GSK3 $\beta$ fluorescence in the cytoplasm compared with cells expressing GFP in WT mice (Fig. 2A). Quantification of GSK3 $\beta$ immunoreactivity revealed significantly lower levels of GSK3 $\beta$ in the nucleus accumbens of GSK3 $\beta$ flx/flx mice injected with AAVCre-GFP compared with WT controls injected with the same vector or GSK3 $\beta$ flx/flx mice injected with AAV-GFP $\left(F_{2,85}=\right.$ 166.3, $P<0.0001$ ) (Fig. 2C). Immunofluorescence analysis of 

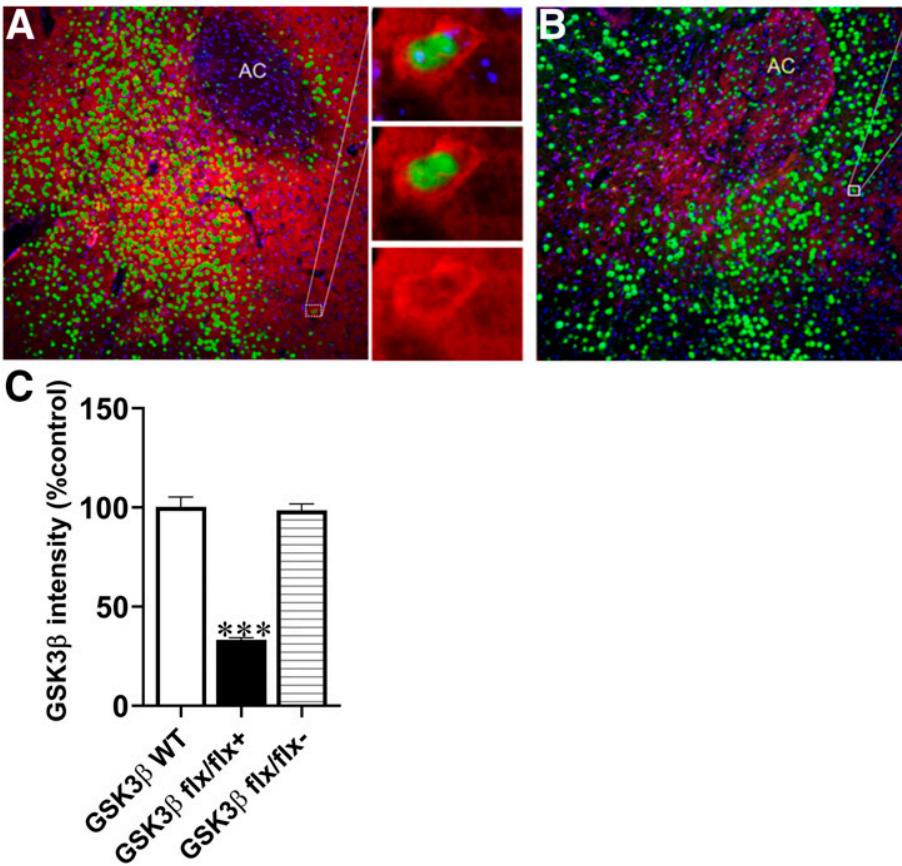

accumbens injected with AAV-Cre-GFP showed a mean $65.85 \%(51 \%-77 \%)$ reduction in GSK3 $\beta$-IR in the GSK3 $\beta \mathrm{flx} /$ flx mice compared with GSK3 $\beta$ WT littermates (Fig. 2C). These data demonstrate effective knockdown of GSK3 $\beta$ in the nucleus accumbens of GSK3 $\beta$ flx/flx mice by AAV-Cre recombinase delivery.

GSK3 $\beta$ Knockdown in the Nucleus Accumbens Attenuated the Development of Cocaine-Induced Conditioned Place Preference. The role of GSK3 $\beta$ in the nucleus accumbens in cocaine conditioned reward was evaluated in conditional GSK3 $\beta$ knockout mice and controls. Four weeks following AAV-Cre injection, when Cre recombination is maximal (Kaspar et al., 2002; Ahmed et al., 2004), cocaine-induced conditioned place preference was performed. One-way ANOVA of preference score revealed a significant difference among the three groups $\left(F_{2,34}=12.64, P<0.0001\right)$ (Fig. 3A). Post-hoc analysis showed that GSK3 $\beta$ flx/flx mice injected with AAVCre-GFP into the accumbens had significantly lower preference scores compared with WT mice injected with AAV-Cre-GFP $(P<0.001)$ and GSK3 $\beta$ flx/flx mice injected with AAV-GFP $(P<$ 0.01 ). Thus, knockdown of GSK3 $\beta$ in the nucleus accumbens significantly impaired the development of cocaine-induced place preference. In Fig. 3B, the correlation between preference score and accumbal GSK3 $\beta$ intensity for individual mice is shown. There was a significant positive correlation between preference scores and the intensity of GSK3 $\beta$ in the nucleus accumbens $\left(r^{2}=0.6353, P=0.0011\right)$, showing that mice with the lowest levels of GSK3 $\beta$ had the lowest preference scores. This finding further supports the contention that activation of GSK $3 \beta$ in the accumbens contributes to cocaine reward. Analysis of the data for potential sex differences (two-way ANOVA with sex and genotype factors) did not reveal a significant difference between males and females, albeit the study may have been underpowered to detect sex differences.

GSK3 $\beta$ Knockdown in the Nucleus Accumbens Had No Effect on the Development of Morphine-Induced Conditioned Place Preference. The requirement of

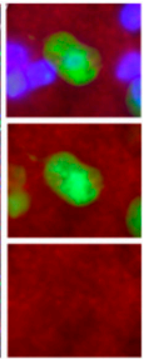

Fig. 2. Representative images show immunostaining of GFP (green), GSK3 $\beta$ (red), and 4,6-diamidino-2-phenylindole dihydrochloride (DAPI) (blue) in the nucleus accumbens of WT (A) and GSK3 $\beta$ flx/flx (B) mice. Immunostaining demonstrates proper targeting of the nucleus accumbens and reductions in the levels of GSK3 $\beta$ protein in GSK3 $\beta \mathrm{flx} /$ flx mice (B, inset). (A) GFP is expressed in cells of the WT accumbens, along with GSK3 $\beta(10 \times)$. Higher magnification $(40 \times)$ shows overlap of GFP and DAPI in an individual cell with intense GSK3 $\beta$ staining in the surrounding cytoplasm. (B) GFP is expressed in cells of the GSK3 $\beta$ flx/flx accumbens $(10 \times)$. Cells that express GFP do not express GSK $3 \beta$ as shown in higher magnification $(40 \times)$. (C) Quantification of GSK3 $\beta$ fluorescence in the nucleus accumbens of WT and GSK3 $\beta \mathrm{flx} / \mathrm{flx}$ mice injected with AAV-Cre-GFP (flx/flx + ) or AAV-GFP (flx/flx-) shows significant reduction of GSK3 $\beta$ following AAV-Cre-GFP injection into the accumbens of GSK3 $\beta$ flx/flx mice. Data are represented as mean \pm S.E.M. $N=20-33 /$ group; AC, anterior commissure. accumbal GSK3 $\beta$ in morphine conditioned reward was tested to investigate the generalizability of the aforementioned findings to another class of drugs. Three weeks after injection of AAV-Cre-GFP into the nucleus accumbens, mice underwent 4-day morphine ( $5 \mathrm{mg} / \mathrm{kg}$ ) conditioning followed by a test for place preference. As shown in Fig. 4A, no significant difference was found in preference scores between GSK3 $\beta$ flx/flx and WT mice with AAV-Cre injections into the accumbens $(t=1.01$, df $=38, P>0.05)$. No significant correlation was found between morphine place preference scores and accumbal GSK3 $\beta$ intensity $\left(r^{2}=0.0039, P=0.80\right)$. To further investigate a potential role of accumbal GSK3 $\beta$ in
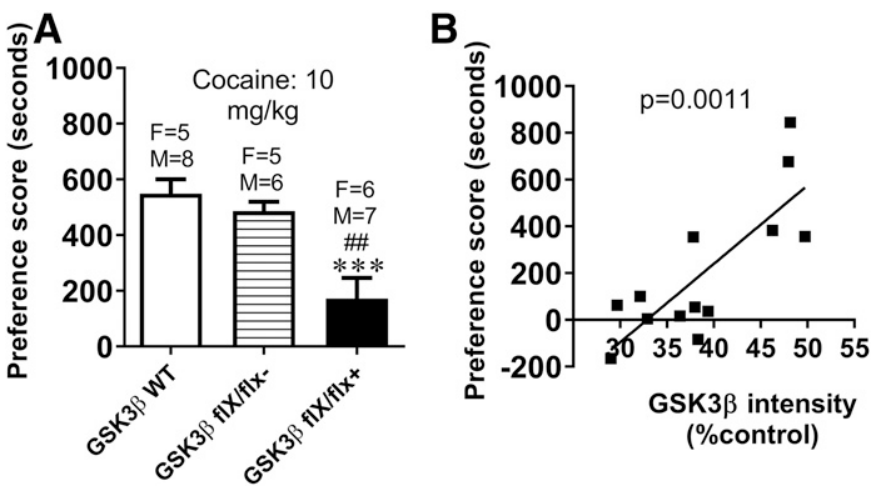

Fig. 3. GSK3 $\beta$ knockdown in the nucleus accumbens attenuates the rewarding effect of cocaine. (A) Conditioned place preference scores are shown for WT mice injected with AAV-Cre, GSK3 $\beta$ flx/flx with AAV-GFP, and GSK $3 \beta \mathrm{flx} / \mathrm{flx}$ with AAV-Cre conditioned with $10 \mathrm{mg} / \mathrm{kg}$ of cocaine. Knockdown of GSK3 $\beta$ in the GSK3 $\beta$ flx/flx + AAV-Cre mice resulted in significantly lower preference scores. Data are expressed as mean + S.E.M. (B) Cocaine preference scores and GSK3 $\beta$ intensity for individual GSK3 $\beta$ flx/flx + mice are shown. Pearson correlation analysis showed a significant positive correlation of preference score and GSK3 $\beta$ intensity in nucleus accumbens of GSK3 $\beta$ flx/flx + mice $\left(r^{2}=0.6353, P=0.0011\right)$. $* * * P<0.001$, GSK3 $\beta$ flx/flx-AAV-Cre vs. WT mice; \#\#P<0.01, GSK3 $\beta$ flx/ flx-AAV-Cre vs. GSK3 $\beta$ flx/flx-AAV-GFP. $N=11-13 /$ group as indicated in the figure; $\mathrm{F}$, female; $\mathrm{M}$, male. 

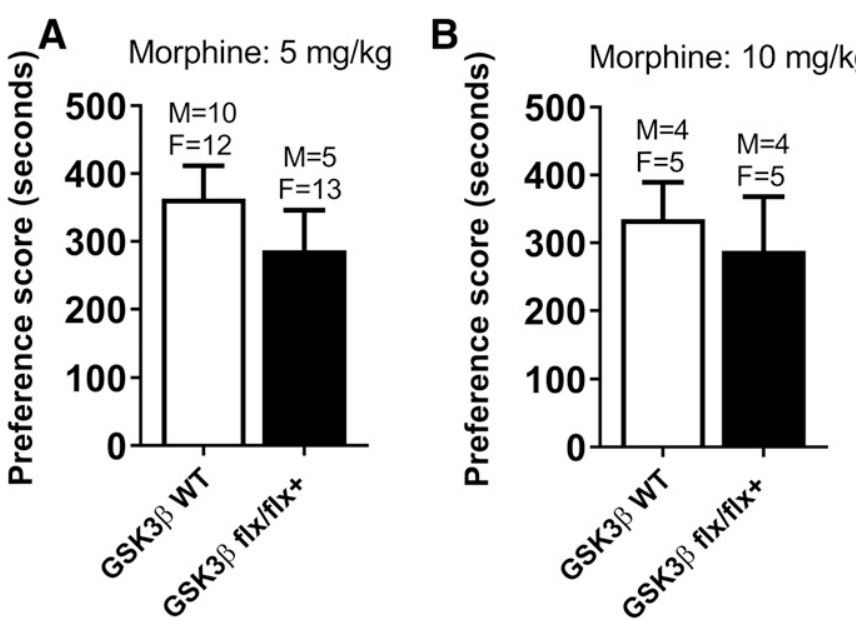

Fig. 4. Morphine conditioned place preference was not changed by GSK3 $\beta$ knockdown in the nucleus accumbens. Conditioned place preference was established with $5 \mathrm{mg} / \mathrm{kg}$ (A) or $10 \mathrm{mg} / \mathrm{kg}$ (B) of morphine in WT mice and GSK3 $\beta \mathrm{flx} / \mathrm{fl}$. No significant differences in preference scores were found between the two groups at either dose. Data are expressed as mean + S.E.M. $N=18-22$ /group.

morphine conditioned reward, another dose of morphine was tested, $10 \mathrm{mg} / \mathrm{kg}$. As shown in Fig. 4B, no significant difference was found in preference scores between GSK3 $\beta$ flx/flx and WT mice with AAV-Cre injections into the accumbens. No significant differences in preference scores were found between males and females of either genotype at any dose of morphine. These data suggest that GSK3 $\beta$ activation in the nucleus accumbens is not required for morphine conditioned reward.

GSK3 $\beta$ Knockdown in the Nucleus Accumbens Attenuated the Development of U50,488H-Induced Conditioned Place Aversion. Place conditioning can assess not only the rewarding but also the aversive effects of drugs. Conditioned place aversion to U50,488H, a $\kappa$-opioid receptor agonist, was performed in GSK3 $\beta$ flx/flx and WT mice 4 weeks after the injection of AAV-Cre-GFP in the nucleus accumbens. As shown in Fig. 5, one-way ANOVA of preferences scores revealed a significant difference between groups $\left(F_{2,27}=6.06\right.$, $P<0.001$ ). Post-hoc analysis demonstrated that GSK3 $\beta \mathrm{flx} /$ flx mice with intra-accumbens injection of AAV-Cre showed significantly less place aversion compared with the WT mice with AAV-Cre $(P<0.05)$ or GSK3 $\beta$ flx/flx mice with control vector injection $(P<0.05)$. No significant differences were observed between males and females in preference scores. There was a significant negative correlation between preference scores and the intensity of GSK3 $\beta$ in the nucleus accumbens $\left(r^{2}=0.48, P=0.03\right)$, indicating that mice with the lowest levels of GSK3 $\beta$ had the least place aversion. These data indicate that GSK3 $\beta$ in nucleus accumbens is required for the development of U50,488H-induced place aversion.

GSK3 $\beta$ Knockdown in the Nucleus Accumbens Did Not Impair Performance in the Novel Object Recognition Task. The novel object recognition task was used to investigate the role of GSK $3 \beta$ in cognitive function. As shown in Fig. 6A, no preference for either object was found for any of the experimental groups during the 10-minute familiarization session $(P>0.05)$. Thirty minutes after the familiarization training, mice underwent a 10 -minute recognition test. Twoway ANOVA of exploration time during the test session revealed a significant main effect of object $\left(F_{1,54}=69.4, P<0.0001\right)$ and

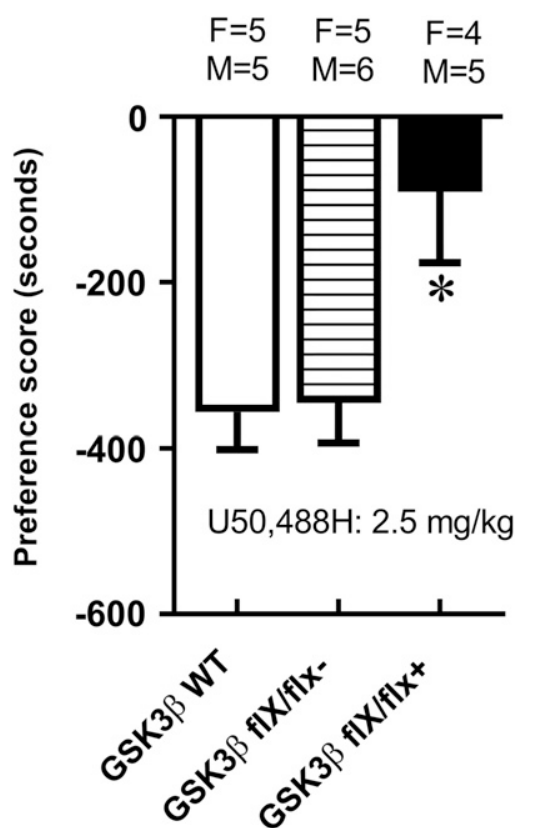

Fig. 5. GSK3 $\beta$ knockdown in nucleus accumbens attenuated the aversive effect of U50,488H. Conditioned place aversion was established with $2.5 \mathrm{mg} / \mathrm{kg}$ of U50,488H. Place aversion scores were significantly reduced in GSK3 $\beta$ flx/flx with AAV-Cre vs. WT mice with AAV-Cre. Data are expressed as mean + S.E.M. ${ }^{*} P<0.05$, GSK3 $\beta$ flx/flx-AAV-Cre vs. WT mice. $N=9-11$ group.

no significant main effect of genotype $\left(F_{2,54}=0.3, P>0.05\right)$ (Fig. 6B). All three experimental groups showed a significant preference for exploring the novel object as shown by the discrimination index $(P<0.001)$ (Fig. $6 \mathrm{C})$. Consistent with the strong preference for novel relative to familiar objects, the DI of the three groups was $45.97 \pm 3.72$ for WT with AAV-Cre, 36.30 \pm 2.91 for GSK3 $\beta$ flx/flx with AAV-GFP, and $38.65 \pm 4.44$ for GSK3 $\beta$ flx/flx with AAV-Cre. No group differences in total exploration times during training or testing were found (data not shown). Likewise, there were no significant differences between males and females in their performance on the novel object recognition task. No significant correlation was observed between DI and accumbal GSK3 $\beta$ intensity $\left(r^{2}=0.0082, P=0.45\right)$. These data show that novel object recognition remains intact in mice with knockdown of GSK3 $\beta$ in the nucleus accumbens.

GSK3 $\beta$ Knockdown in the Nucleus Accumbens Impaired Performance in the Novel Location Task. Mice underwent the object location task, whereby they were trained with two identical objects for in the familiarization session. Thirty minutes following the familiarization session, mice were placed in the same arena with one object located in a new position. As shown in Fig. 7A, during the familiarization session, no preference for the object position was found in WT mice $(P>0.05)$, GSK3 $\beta$ flx/flx mice with intra-accumbens injection of AAV-Cre $(P>0.05)$, or GSK3 $\beta$ flx/flx mice with AAV-GFP infusion $(P>0.05)$. During the test phase (Fig. 7B), two-way ANOVA of exploration time revealed a significant main effect of location $\left(F_{1,54}=31.2, P<0.001\right)$ and significant location $\times$ genotype interaction $\left(F_{2,54}=5.3, P<0.01\right)$, but no significant main effect of genotype $\left(F_{1,54}=1.9, P>\right.$ $0.05)$. Post-hoc analysis showed that WT mice spent more time with the object in the novel location $(P<0.001)$, thus exhibiting memory for the familiar object location, as did GSK3 $\beta$ flx/flx mice with control vector infusion $(P<0.001)$. In contrast, time 

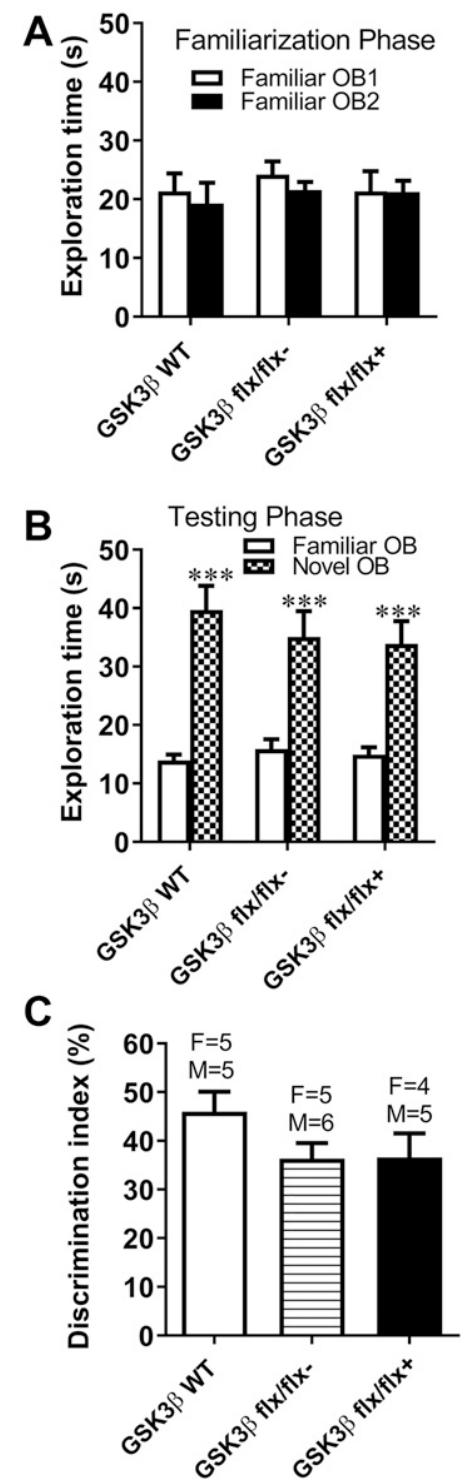

Fig. 6. Novel object recognition task remains intact in mice with GSK- $3 \beta$ knockdown in nucleus accumbens. WT, GSK3 $\beta$ flx/flx with AAV-Cre, and GSK3 $\beta$ flx/flx with AAV-GFP mice underwent novel object recognition testing. (A) There was no innate preference for either object during the familiarization phase. (B) All groups explored the novel object more than the familiar object during the testing phase. (C) No significant differences in the DI were found. Data are expressed as mean + S.E.M. ***P $<0.001$ vs. familiar object (OB). $N=9-11$.

spent in exploring the objects did not differ between the familiar and novel locations in GSK3 $\beta$ flx/flx mice with knockdown of GSK3 $\beta$ in the accumbens. The DI was significantly lower in mice with accumbal GSK3 $\beta$ knockdown compared with control WT mice $(P<0.001)$ or control GSK3 $\beta$ flx/flx mice $(P<0.01)$, as shown in Fig. 7C. No differences in total exploration times between groups during training or testing were found. Analysis of males and females separately by two-way ANOVA with sex and genotype factors revealed no significant sex differences. There was no significant correlation between the DI and accumbal GSK3 $\beta$ intensity $\left(r^{2}=0.12, P=0.35\right)$. These data demonstrate that GSK3 $\beta$ knockdown in nucleus accumbens produced a cognitive deficit in location memory in male and female mice.

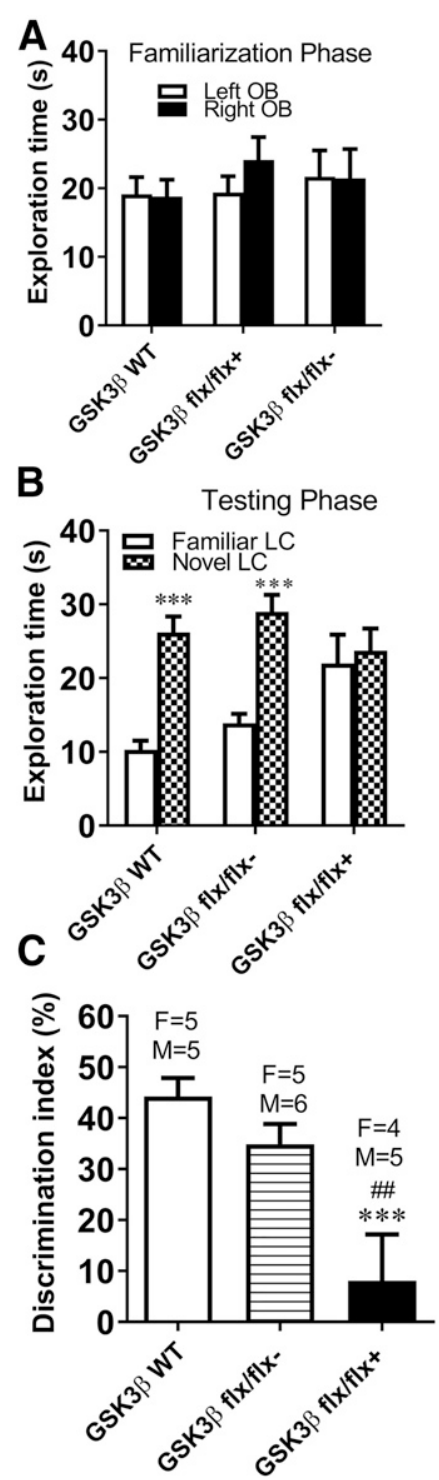

Fig. 7. Performance in the novel location task was impaired in mice with conditional knockdown of GSK3 $\beta$ in the nucleus accumbens. (A) No innate preference for object position was found in the three groups of mice during the familiarization phase. (B) WT mice with AAV-Cre and GSK3 $\beta$ flx/flx mice with AAV-GFP, but not the mice with GSK3 $\beta$ knockdown in the nucleus accumbens, spent more time exploring the object in a new location than the prior location. $* * * P<0.001$ vs. familiar object (OB). (C) GSK3 $\beta$ knockdown significantly reduced the DI compared with controls, indicating impairment in special memory. $* * * P<0.001$ vs. WT mice with AAV-Cre; \#\#P $<0.01$ vs. GSK3 $\beta$ flx/flx mice with AAV-GFP. Data are expressed as mean + S.E.M. $N=9-11$ /group.

\section{Discussion}

This study elucidated the role of GSK3 $\beta$ specifically within the nucleus accumbens in drug reward and aversion and object and spatial memory tasks. Prior work using systemically administered GSK3 inhibitors has demonstrated the importance of GSK3 in drug reward and memory (Wu et al., 2011; Miller et al., 2014; Shi et al., 2014; Wickens et al., 2017). GSK3 $\beta$ is widely expressed throughout the brain, but the region(s) where GSK3 $\beta$ influences these functions is largely unknown. Knockdown of accumbal GSK3 $\beta$ in floxed mice impaired their ability to develop a cocaine-induced place preference. Further analysis revealed a significant positive 
correlation between nucleus accumbens GSK3 $\beta$ levels and cocaine preference scores. Mice with low GSK3 $\beta(<40 \%$ of control) showed no preference for the cocaine-paired environment, supporting the importance of GSK3 $\beta$ specifically in the nucleus accumbens in mediating cocaine-induced place preference. In contrast, mice with reduced accumbens GSK3 $\beta$ showed a significant preference for the morphine-paired environment similar to WT mice. The ability of the GSK3 $\beta$ knockdown mice to develop and express a morphine place preference supports the contention that cocaine reward processes were affected by the knockdown rather than an inability of the mice to learn or remember the place conditioning task. Thus, GSK3 $\beta$ signaling in the accumbens is a critical component of positive rewarding actions of cocaine, but is not necessary for morphine conditioned reward.

Dopaminergic neurons of the ventral tegmental area (VTA) projecting to the nucleus accumbens comprise the primary pathway mediating the reinforcing effects of drugs of abuse and natural rewards (Wise, 1996; Koob and Le Moal, 1997). Despite its central role in reward processes, actions of opiates versus stimulants on this circuit differ (Koo et al., 2012) . For example, opiates and stimulants regulate structural plasticity in opposing manners. Opiates decrease the number and complexity of dendritic spines on accumbens medium spiny neurons (MSNs), as well as the size of VTA dopamine neurons (Sklair-Tavron et al., 1996; Robinson and Kolb, 2004; Russo et al., 2007), whereas stimulants increase MSN dendritic complexity and spine density (Robinson et al., 2001; Norrholm et al., 2003; Lee et al., 2006; Sarti et al., 2007) and increase VTA dopaminergic neuronal size (Sarti et al., 2007). Other evidence suggests that morphine and cocaine may induce conditioned place preference through different physiologic mechanisms. German and Fields (2007) recorded neurons in the nucleus accumbens of rats during the expression of morphine-induced place preference and found that MSNs slowed firing rates when the rat was in the morphine-paired zone. In contrast, Sjulson et al. (2018) found that cocaineinduced place preference was associated with recruitment of D2-positive MSNs to fire in the cocaine-paired location. Thus, cocaine and morphine interact with the VTA-accumbens pathway in unique ways. The present results show the different reliances of cocaine versus morphine on GSK3 $\beta$ signaling in the nucleus accumbens in the development of drug-associated place preference. Although the accumbens is clearly a site of importance, further studies are needed to identify neuron-specific contributions of GSK $3 \beta$, and GSK $3 \beta$ in other brain regions may work independently or in concert with the accumbens to mediate cocaine reward.

GSK3 $\beta$ in cocaine self-administration has been reported (Crofton et al., 2017). GSK3 $\beta$ was reduced by $35 \%$ in the medial shell of the accumbens by delivery of AAV-shGSK3 $\beta$ in the rat. GSK $3 \beta$ knockdown did not affect cocaine acquisition, progressive ratio responding, extinction, or cocaine-primed reinstatement behaviors. Rats with reduced GSK3 $\beta$ in the accumbens medial shell showed a small but significant increase in responding at high unit doses of cocaine, but no differences at lower doses (Crofton et al., 2017). It is difficult to compare self-administration and place conditioning models given the different processes involved in operant responding versus Pavlovian condition, as well as the level of drug exposure (high drug exposure in self-administration vs. low drug exposure in place conditioning). Furthermore, the larger reduction in GSK3 $\beta$ in both the accumbens core and shell regions that was achieved in the current study could have a greater influence on cocaine reward than the limited reductions in GSK3 $\beta$ in the medial shell in the aforementioned study, given the known differences in the role of core versus shell in reward processes (e.g., West and Carelli, 2016). These procedural differences (e.g., extent and location of GSK3 $\beta$ knockdown, cocaine dose, and species) need to be rigorously tested to make firm conclusions about GSK3 $\beta$ in cocaine conditioned reward versus self-administration.

Like conditioned place preference, conditioned place aversion involves similar Pavlovian processes, but with a negative component such that animals avoid a context previously associated with an aversive state. The activation of $\kappa$-opioid receptors produces a negative state associated with dysphoria and anxiety (Lalanne et al., 2014), and thus subjects given a $\kappa$ opioid receptor agonist in a specific environment show a conditioned aversion to that environment (Shippenberg and Herz, 1986). Motivational systems in the nucleus accumbens are necessary not only for obtaining rewards but also for the avoidance of harm (Kohls et al., 2013; Wise and Koob, 2014; Wenzel et al., 2015). Previous work has indicated that dopaminergic transmission in the accumbens is required for the $\kappa$-opioid receptor agonist U50,488H to produce conditioned place aversion (Bals-Kubik et al., 1993). Furthermore, intraaccumbens infusion of the D1-receptor antagonist $\mathrm{R}(+)-7$ chloro-8-hydroxy-3-methyl-1-phenyl-2,3,4,5-tetrahydro-1H-3benzazepine hydrochloride ( $\mathrm{SCH} 23390$ ) blocks the acquisition of $\kappa$-receptor agonist-induced place aversion (Shippenberg et al., 1993). The present results demonstrate that knockdown of GSK3 $\beta$ interfered with the development of U50,488Hinduced place aversion, indicating that accumbal GSK3 $\beta$ signaling plays an important role in the negative state produced by $\kappa$-receptor agonists. A recent report demonstrated that subpopulations of mesolimbic dopamine neurons respond differently to appetitive and aversive stimuli (de Jong et al., 2019). Excitation to reward-predicting cues dominates in accumbens lateral shell dopamine terminals, whereas aversive prediction signals in ventral accumbens medial shell (de Jong et al., 2019). Other results support the conclusion that the nucleus accumbens is involved in both reward and aversive learning but indicates that reward-based behaviors are dependent on transmission of the direct striatonigral pathway, whereas aversive learning behaviors are dependent on indirect striatopallidal transmission (Hikida et al., 2010). Further studies are needed to establish the roles of GSK3 $\beta$ in different subregions and cell populations of the accumbers in both cocaine reward and U50,488H-induced conditioned aversion.

The novel object recognition test is used to detect both impairment and enhancement of memory. A short delay (0.5-3 hours) between training and testing phases is used for studying memory impairment, and a longer delay ( 24 hours) is used for testing memory improvement (Papp et al., 2017). The current study used a 30-minute delay interval after object training (familiarization) to test for memory deficits in mice with accumbal GSK3 $\beta$ knockdown. The results demonstrated intact novel object memory; however, performance on the object location task was impaired by GSK3 $\beta$ knockdown. Thus, differential roles of accumbal GSK3 $\beta$ exist between memory for object identity and the respective spatial location. Different brain regions contribute to performance of the novel 
object recognition and object location tasks. The insular, perirhineal, and medial prefrontal cortices contribute to the learning and memory formation of object recognition (Mendez et al., 2015; Tanimizu et al., 2018). Object location recognition is dependent on spatial learning and memory. The role of hippocampus in novel object recognition and/or location memory formation is controversial. Several studies have shown that the hippocampus is required for object location memory but not novel object recognition memory (Mumby et al., 2002; Balderas et al., 2008; Roozendaal et al., 2010), especially in relation to one-trial object recognition tasks (Forwood et al., 2005) such as those used in the current study. The nucleus accumbens contributes to the processing of hippocampaldependent spatial information (Ferretti et al., 2005), and performance on spatial tasks depends on functional interaction between the hippocampus and accumbens (Ito et al., 2008). Neuroanatomical evidence has demonstrated that the accumbens medial shell receives input from the ventral hippocampus, whereas the core and rostrolateral shell are innervated largely by the dorsal hippocampus (Groenewegen et al., 1999). Other work has shown that the core and shell of the nucleus accumbens and their dopamine innervation are involved in different forms of recognition memory (Nelson et al., 2010). For example, dopamine depletion in the core or the core plus the shell, but not the shell alone, disrupts novel object memory after a 24-hour delay, but not after a short (5minute) delay. In contrast, dopamine depletion in the shell or a larger shell plus core lesions impaired short-term location memory (10-minute delay) in the object location test (Nelson et al., 2010). These evidences support our present finding that knockdown of GSK3 $\beta$ in the nucleus accumbens (core plus shell) affects short-term spatial location memory, but not nonspatial object memory.

In summary, these data demonstrate the importance of GSK3 $\beta$ in the nucleus accumbens for the development of both cocaine conditioned place preference and U50,488H conditioned place aversion, but not for morphine place preference. Moreover, GSK3 $\beta$ signaling in the nucleus accumbens engages during the object location task, but not in the novel object recognition task. Thus, GSK3 $\beta$ in the nucleus accumbens plays differential roles in cocaine and opiate reward process, as well as in memory for object identity and the respective spatial location.

\section{Acknowledgments}

We acknowledge Dr. Jim R. Woodgett from the LunenfeldTanenbaum Research Institute in Toronto, Canada, for generously providing the GSK3 $\beta$ floxed mice used to establish our breeding colony. We also thank Ahmed Mirza and Michael Zaykaner for contributing to the successful completion of this study. We are grateful to the National Institute on Drug Abuse drug supply program for the generous contribution of the drugs used in this study.

\section{Authorship Contributions}

Participated in research design: Shi, Barr, Unterwald.

Conducted experiments: Shi, Barr, von Weltin, Wolsh.

Contributed new reagents or analytic tools: Shi, Barr.

Performed data analysis: Shi, Barr.

Wrote or contributed to the writing of the manuscript: Shi, Barr, Unterwald, von Weltin, Wolsh

\section{References}

Ahmed BY, Chakravarthy S, Eggers R, Hermens WT, Zhang JY, Niclou SP, Levelt C, Sablitzky F, Anderson PN, Lieberman AR, et al. (2004) Efficient delivery of
Cre-recombinase to neurons in vivo and stable transduction of neurons using adeno-associated and lentiviral vectors. BMC Neurosci 5:4.

Balderas I, Rodriguez-Ortiz C.J, Salgado-Tonda P, Chavez-Hurtado J, McGaugh JL, and Bermudez-Rattoni $\mathrm{F}$ (2008) The consolidation of object and context recognition memory involve different regions of the temporal lobe. Learn Mem 15:618-624.

Bals-Kubik R, Ableitner A, Herz A, and Shippenberg TS (1993) Neuroanatomica sites mediating the motivational effects of opioids as mapped by the conditioned place preference paradigm in rats. J Pharmacol Exp Ther 264:489-495.

Beaulieu JM, Del'guidice T, Sotnikova TD, Lemasson M, and Gainetdinov RR (2011) Beyond cAMP: the regulation of Akt and GSK3 by dopamine receptors. Front Mol Neurosci 4:38.

Beaulieu JM, Sotnikova TD, Marion S, Lefkowitz RJ, Gainetdinov RR, and Caron MG (2005) An Akt/ $\beta$-arrestin 2/PP2A signaling complex mediates dopaminergic neurotransmission and behavior. Cell 122:261-273.

Beaulieu JM, Sotnikova TD, Yao WD, Kockeritz L, Woodgett JR, Gainetdinov RR, and Caron MG (2004) Lithium antagonizes dopamine-dependent behaviors mediated by an AKT/glycogen synthase kinase 3 signaling cascade. Proc Natl Acad Sci USA 101:5099-5104.

Coghlan MP, Culbert AA, Cross DA, Corcoran SL, Yates JW, Pearce NJ, Rausch OL, Murphy GJ, Carter PS, Roxbee Cox L, et al. (2000) Selective small molecule inhibitors of glycogen synthase kinase-3 modulate glycogen metabolism and gene transcription. Chem Biol 7:793-803.

Crofton EJ, Nenov MN, Zhang Y, Scala F, Page SA, McCue DL, Li D, Hommel JD, Laezza F, and Green TA (2017) Glycogen synthase kinase 3 beta alters anxiety-, depression-, and addiction-related behaviors and neuronal activity in the nucleus accumbens shell. Neuropharmacology 117:49-60.

de Jong JW, Afjei SA, Pollak Dorocic I, Peck JR, Liu C, Kim CK, Tian L, Deisseroth $\mathrm{K}$, and Lammel S (2019) A neural circuit mechanism for encoding aversive stimuli in the mesolimbic dopamine system. Neuron 101:133-151.e7.

Ferretti V, Florian C, Costantini VJ, Roullet P, Rinaldi A, De Leonibus E, Oliverio A and Mele A (2005) Co-activation of glutamate and dopamine receptors within the nucleus accumbens is required for spatial memory consolidation in mice. Psychopharmacology (Berl) 179:108-116.

Forwood SE, Winters BD, and Bussey TJ (2005) Hippocampal lesions that abolish spatial maze performance spare object recognition memory at delays of up to 48 hours. Hippocampus 15:347-355.

German PW and Fields HL (2007) Rat nucleus accumbens neurons persistently encode locations associated with morphine reward. J Neurophysiol 97:2094-2106.

Gould TD, Zarate CA, and Manji HK (2004) Glycogen synthase kinase-3: a target for novel bipolar disorder treatments. J Clin Psychiatry 65:10-21.

Grimes CA and Jope RS (2001) CREB DNA binding activity is inhibited by glycogen synthase kinase-3 $\beta$ and facilitated by lithium. J Neurochem 78:1219-1232.

Groenewegen HJ, Wright CI, Beijer AV, and Voorn P (1999) Convergence and segregation of ventral striatal inputs and outputs. Ann N Y Acad Sci 877:49-63.

Hikida T, Kimura K, Wada N, Funabiki K, and Nakanishi S (2010) Distinct roles of synaptic transmission in direct and indirect striatal pathways to reward and aversive behavior. Neuron 66:896-907.

Ito R, Robbins TW, Pennartz CM, and Everitt BJ (2008) Functional interaction between the hippocampus and nucleus accumbens shell is necessary for the acquisition of appetitive spatial context conditioning. J Neurosci 28:6950-6959.

Jope RS and Johnson GV (2004) The glamour and gloom of glycogen synthase kinase3. Trends Biochem Sci 29:95-102.

Kaspar BK, Vissel B, Bengoechea T, Crone S, Randolph-Moore L, Muller R, Brandon EP, Schaffer D, Verma IM, Lee KF, et al. (2002) Adeno-associated virus effectively mediates conditional gene modification in the brain. Proc Natl Acad Sci USA 99: $2320-2325$

Kimura T, Yamashita S, Nakao S, Park JM, Murayama M, Mizoroki T, Yoshiike Y, Sahara N, and Takashima A (2008) GSK- $3 \beta$ is required for memory reconsolidation in adult brain. PLoS One $3: \mathrm{e} 3540$.

Kockeritz L, Doble B, Patel S, and Woodgett JR (2006) Glycogen synthase kinase-3an overview of an over-achieving protein kinase. Curr Drug Targets 7:1377-1388

Kohls G, Perino MT, Taylor JM, Madva EN, Cayless SJ, Troiani V, Price E, Faja S, Herrington JD, and Schultz RT (2013) The nucleus accumbens is involved in both the pursuit of social reward and the avoidance of social punishment. Neuropsychologia 51:2062-2069.

Koo JW, Mazei-Robison MS, Chaudhury D, Juarez B, LaPlant Q, Ferguson D, Feng J, Sun H, Scobie KN, Damez-Werno D, et al. (2012) BDNF is a negative modulator of morphine action. Science 338:124-128.

Koob GF and Le Moal M (1997) Drug abuse: hedonic homeostatic dysregulation. Science 278:52-58

Lalanne L, Ayranci G, Kieffer BL, and Lutz PE (2014) The kappa opioid receptor: from addiction to depression, and back. Front Psychiatry $\mathbf{5}: 170$.

Lee KW, Kim Y, Kim AM, Helmin K, Nairn AC, and Greengard P (2006) Cocaineinduced dendritic spine formation in D1 and D2 dopamine receptor-containing medium spiny neurons in nucleus accumbens. Proc Natl Acad Sci USA 103 3399-3404

Liu JJ, Chiu YT, DiMattio KM, Chen C, Huang P, Gentile TA, Muschamp JW, Cowan A, Mann M, and Liu-Chen LY (2019) Phosphoproteomic approach for agonistspecific signaling in mouse brains: mTOR pathway is involved in $\kappa$ opioid aversion. Neuropsychopharmacology 44:939-949.

Lovestone S, Killick R, Di Forti M, and Murray R (2007) Schizophrenia as a GSK-3 dysregulation disorder. Trends Neurosci 30:142-149.

Mendez M, Arias N, Uceda S, and Arias JL (2015) c-Fos expression correlates with performance on novel object and novel place recognition tests. Brain Res Bull 117 $16-23$

Miller JS, Barr JL, Harper LJ, Poole RL, Gould TJ, and Unterwald EM (2014) The GSK3 signaling pathway is activated by cocaine and is critical for cocaine conditioned reward in mice. PLoS One 9:e88026.

Mumby DG, Gaskin S, Glenn MJ, Schramek TE, and Lehmann H (2002) Hippocampal damage and exploratory preferences in rats: memory for objects, places, and contexts. Learn Mem 9:49-57. 
Nelson AJ, Thur KE, Marsden CA, and Cassaday HJ (2010) Dissociable roles of dopamine within the core and medial shell of the nucleus accumbens in memory for objects and place. Behav Neurosci 124:789-799.

Norrholm SD, Bibb JA, Nestler EJ, Ouimet CC, Taylor JR, and Greengard P (2003) Cocaine-induced proliferation of dendritic spines in nucleus accumbens is dependent on the activity of cyclin-dependent kinase-5. Neuroscience 116 : $19-22$.

Papp M, Gruca P, Lason-Tyburkiewicz M, Litwa E, Niemczyk M, Tota-Glowczyk K and Willner P (2017) Dopaminergic mechanisms in memory consolidation and antidepressant reversal of a chronic mild stress-induced cognitive impairment. Psychopharmacology (Berl) 234:2571-2585.

Patel S, Doble BW, MacAulay K, Sinclair EM, Drucker DJ, and Woodgett JR (2008) Tissue-specific role of glycogen synthase kinase $3 \beta$ in glucose homeostasis and insulin action. Mol Cell Biol 28:6314-6328.

Paxinos G and Franklin KBJ (2001) The Mouse Brain in Stereotaxic Coordinates, Academic Press, San Diego, CA.

Perrine SA, Miller JS, and Unterwald EM (2008) Cocaine regulates protein kinase B and glycogen synthase kinase-3 activity in selective regions of rat brain. $J$ Neurochem 107:570-577.

Robinson TE, Gorny G, Mitton E, and Kolb B (2001) Cocaine self-administration alters the morphology of dendrites and dendritic spines in the nucleus accumbens and neocortex. Synapse 39:257-266.

Robinson TE and Kolb B (2004) Structural plasticity associated with exposure to drugs of abuse. Neuropharmacology 47 (Suppl 1):33-46.

Roozendaal B, Hernandez A, Cabrera SM, Hagewoud R, Malvaez M, Stefanko DP, Haettig J, and Wood MA (2010) Membrane-associated glucocorticoid activity is necessary for modulation of long-term memory via chromatin modification. $J$ Neurosci 30:5037-5046.

Russo SJ, Bolanos CA, Theobald DE, DeCarolis NA, Renthal W, Kumar A Winstanley CA, Renthal NE, Wiley MD, Self DW, et al. (2007) IRS2-Akt pathway in midbrain dopamine neurons regulates behavioral and cellular responses to opiates. Nat Neurosci 10:93-99.

Sarti F, Borgland SL, Kharazia VN, and Bonci A (2007) Acute cocaine exposure alters spine density and long-term potentiation in the ventral tegmental area. Eur $J$ Neurosci 26:749-756.

Shi X, Miller JS, Harper LJ, Poole RL, Gould TJ, and Unterwald EM (2014) Reactivation of cocaine reward memory engages the Akt/GSK3/mTOR signaling pathway and can be disrupted by GSK3 inhibition. Psychopharmacology (Berl) 231: 3109-3118.

Shippenberg TS, Bals-Kubik R, and Herz A (1993) Examination of the neurochemical substrates mediating the motivational effects of opioids: role of the mesolimbic dopamine system and D-1 vs. D-2 dopamine receptors. J Pharmacol Exp Ther 265: $53-59$

Shippenberg TS and Herz A (1986) Differential effects of mu and kappa opioid systems on motivational processes. NIDA Res Monogr 75:563-566.

Silverman JL, Oliver CF, Karras MN, Gastrell PT, and Crawley JN (2013) AMPAKINE enhancement of social interaction in the BTBR mouse model of autism. Neuropharmacology 64:268-282.

Sjulson L, Peyrache A, Cumpelik A, Cassataro D, and Buzsaki G (2018) Cocaine place conditioning strengthens location-specific hippocampal coupling to the nucleus accumbens. Neuron 98:926-934.e5.

Sklair-Tavron L, Shi WX, Lane SB, Harris HW, Bunney BS, and Nestler EJ (1996) Chronic morphine induces visible changes in the morphology of mesolimbic dopamine neurons. Proc Natl Acad Sci USA 93:11202-11207.

Tanimizu T, Kono K, and Kida S (2018) Brain networks activated to form object recognition memory. Brain Res Bull 141:27-34.

Wenzel JM, Rauscher NA, Cheer JF, and Oleson EB (2015) A role for phasic dopamine release within the nucleus accumbens in encoding aversion: a review of the neurochemical literature. ACS Chem Neurosci 6:16-26.

West EA and Carelli RM (2016) Nucleus accumbens core and shell differentially encode reward-associated cues after reinforcer devaluation. $J$ Neurosci 36: $1128-1139$.

Wickens RH, Quartarone SE, and Beninger RJ (2017) Inhibition of glycogen synthase kinase-3 by SB 216763 affects acquisition at lower doses than expression of amphetamine-conditioned place preference in rats. Behav Pharmacol 28:262-271.

Wise RA (1996) Addictive drugs and brain stimulation reward. Annu Rev Neurosci 19:319-340.

Wise RA and Koob GF (2014) The development and maintenance of drug addiction. Neuropsychopharmacology 39:254-262.

Wu P, Xue YX, Ding ZB, Xue LF, Xu CM, and Lu L (2011) Glycogen synthase kinase $3 \beta$ in the basolateral amygdala is critical for the reconsolidation of cocaine reward memory. $J$ Neurochem 118:113-125.

Yao HB, Shaw PC, Wong CC, and Wan DC (2002) Expression of glycogen synthase kinase-3 isoforms in mouse tissues and their transcription in the brain. $J$ Chem Neuroanat 23:291-297.

Address correspondence to: Dr. Xiangdang Shi, Center for Substance Abuse Research, Lewis Katz School of Medicine at Temple University, 3500 North Broad Street, Philadelphia, PA 19140. E-mail: xiangdang.shi@ temple.edu 